\title{
Revue bibliographique et premières observations en Guadeloupe sur Thrips palmi Karny
}

\author{
Jean GUYOT \\ INRA, Station de Zoologie et de Lutte biologique, Centre de Recherches des Antilles et de la Guyane, B.P. 1232, \\ 97184 Pointe-à-Pitre Cedex
}

RÉSUMÉ

Le présent article regroupe l'ensemble des informations de la littérature actuelle sur Thrips palmi, Karny, important ravageur des cultures d'aubergines et de Cucurbitacées dans les Antilles Françaises. Il présente des données relatives à la répartition géographique et aux plantes hôtes de cette espéce dans le monde. On y trouvera aussi les connaissances actuelles sur sa biologie, sur son écologie et sur les moyens de lutte qui ont été testés ou utilisés dans divers pays. Ces données sont complétées par les premières observations réalisées en Guadeloupe.

Mots clés additionnels : Répartition géographique, plantes hôtes, dégâts, méthode d'élevage, biologie, écologie, dynamique des populations, ennemis naturels, lutte chimique, lutte agronomique.

Additional key words : Distribution, host plants, damage, rearing method, biology, population dynamics, natural ennemies, chemical control, cultural control.

\section{INTRODUCTION}

Depuis son apparition en Guadeloupe en 1985, Thrips palmi Karny a eu une incidence économique désastreuse sur les cultures dont il se nourrit, principalement les Cucurbitacées (melon, concombre) et les Solanacées (aubergine, poivron). Ainsi, les exportations d'aubergine de la Guadeloupe ont chuté de 5000 tonnes en 1985 à 1600 tonnes en 1986. De même, le concombre, qui se trouvait en situation de surproduction s'est brutalement retrouvé en situation de rareté avec un triplement de son prix sur le marché local.

En Martinique, Denoyes et al. (1986) notaient que 37 p. 100 des cultures maraîchères des 2 principales coopératives de l'île étaient sinistrés et que, dans les autres plantations, 90 p. 100 des champs d'aubergine étaient atteints et les exportations réduites de la même proportion. Les cultures de concombre, pastèque et melon étaient également fortement touchées. Les problèmes posés par cet insecte risquent de représenter pour la Guadeloupe un frein important à la politique de diversification agricole qui s'appuie pour une grande part sur les productions maraîchères.

Face à l'apparition brutale de ce fléau, s'imposait la recherche immédiate d'un moyen chimique offrant une riposte rapide au problème. Malheureusement, les essais menés conjointement en 1986 par l'INRA et le GRISP (HOSTACHY et al., 1986a, b, $c, d, e$ ) n'ont permis de mettre en évidence qu'une seule matière active, le profenofos, efficace contre ce ravageur avec les risques de sélection de races résistantes que cela suppose.

Une deuxième phase de recherche a été prise en charge par l'INRA Antilles-Guyane et l'IRAT Martinique afin de mettre au point une méthode de lutte intégrée 
combinant des moyens chimiques, biologiques et agronomiques. Dans ce but, il était nécessaire de faire le point des connaissances actuelles sur cet insecte en consultant les travaux déjà réalisés sur ce sujet. C'est l'ensemble de ces informations que nous regroupons ici, avec quelques compléments apportés par les premières observations en Guadeloupe. La littérature relative à cette question est encore assez réduite et difficile à exploiter puisqu'un grand nombre de publications sont en langue japonaise.

\section{RÉPARTITION GÉOGRAPHIQUE ET PLANTES HÔTES}

Thrips palmi a été décrit pour la première fois en 1925 à Sumatra. Sa présence est connue depuis de nombreuses années dans tout l'est asiatique (BнATTI, 1980; Crowe, 1985), en particulier sur coton en Thailande (WANGBOONKONG, 1981), en Chine et en Inde et sur tabac en Indonésie (Bournier, 1986).

Depuis environ 10 ans, sa répartition géographique s'est largement étendue. En effet, il a été découvert en 1978-1979 aux Philippines sur coton (Bournier, 1983), en Nouvelle-Calédonie et à Wallis sur cultures maraîchères et à La Réunion sur oignon (Bournier. 1986). Sa présence dans l'Océan Indien a été confirmée récemment par VERCAMBRE (comm. pers.) qui signale des dégâts importants sur cultures maraîchères à l'Ile Maurice alors qu'à La Réunion le problème n'a pas encore atteint un niveau économiquement inquiétant. L'apparition de Thrips palmi au Japon sur pomme de terre et sur cultures maraichères date également de 1978. Il occasionne aussi de graves dommages sur cultures maraîchères à Taiwan (WANG et CHU, 1986a, 1987) et sa présence a été constatée à Hawaï en 1984 (Jounson, 1986) sur pastèque en plein champ. Enfin, depuis 1985 , il est responsable de nombreux dégâts en Guadeloupe et en Martinique sur cultures maraîchères (aubergine. concombre, melon, poivron). Son aire de répartition dans la région Caraïbe semble s'étendre rapidement puisqu'il est apparu récemment à Antigua et à Porto Rico (ANAIS, comm. pers.).

Cette espèce est très polyphage et elle peut s'attaquer à un grand nombre de végétaux appartenant à plusieurs familles botaniques. Ainsi, elle peut se nourrir d'une grande quantité de plantes sauvages ou subspontanées et un certain nombre de cultures de moindre importance économique peuvent être l'objet d'attaques parfois virulentes: niébé à Hawaï (JoHnson, 1986) et aux Philippines (Ruhendi \& Litsinger, 1979; Litsinger \& RUHENDI, 1984), sésame en Inde (KUMAR et ANAN'THAKRISHNAN, 1984), haricot aux Antilles Françaises. MESSIAEN (comm. pers.) remarque que parmi les plantes maraîchères hôtes de ce ravageur, les plus attaquées sont celles dont le taux d'accroissement par jour dépasse 0,1 et le taux de reproduction par mois 20 (concombre, melon, aubergine, poivron), ceci aussi bien dans les Antilles Françaises que dans le reste du monde et en particulier au Japon. Des plantes telles que le haricot ou la pomme de terre sont beaucoup moins sensibles. Nos observations en Guadeloupe ont permis de constater que Thrips palmi ne se nourrit pas sur tomate, même occasionnellement, ce qui est confirmé par les essais d'infestation artificielle réalisés par KaWAI (1986a).

\section{SYMPTÔMES DES ATTAQUES \\ ET ÉVALUATION CHIFFRÉE SUR \\ QUELQUES PLANTES MARAÎCHËRES}

\section{A. Sur aubergine}

En début d'attaques, on observe sur les feuilles une coloration nacrée ou argentée qui débute le long des nervures principales. A un stade plus avancé, cette décoloration s'étend à l'ensemble de la feuille. De plus, l'extension du limbe est contrariée chez les jeunes feuilles qui se dessèchent, les plants étant alors fortement diminués (fig. 1). Les attaques de thrips se manifestent aussi par une subérisation de l'épiderme des jeunes fruits (fig. 2): ces zones subérifiées pourront

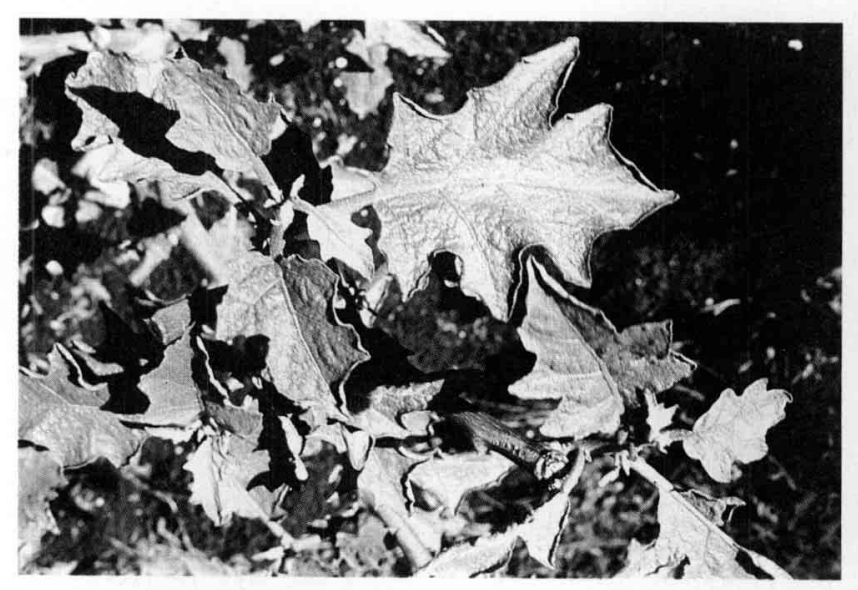

Figure 1

Dégâts de Thrips palmi sur feuilles d'aubergines

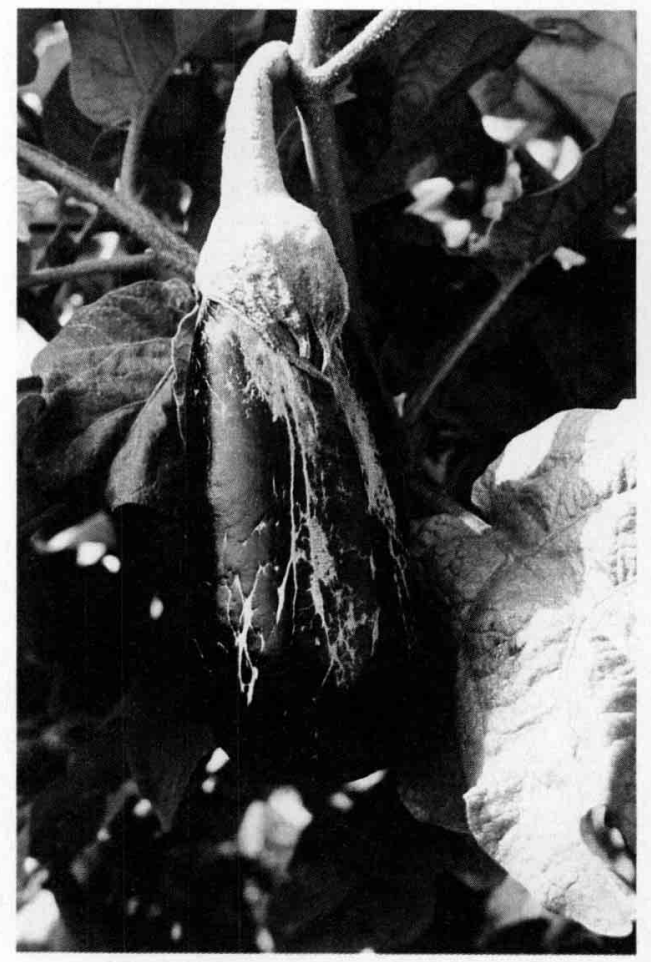

Figure 2

Dégâs de Thrips palmi sur fruits. 
recouvrir 30 à 40 p. 100 de la surface du fruit à maturité, d'où son déclassement ou son refus à la vente (jusqu'à 80 p. 100 des aubergines en Guadeloupe). Les dégâts, surtout latéraux et entourés d'une zone verte, sont à distinguer de ceux dus aux acariens qui sont encore plus subérisés et débutent à la pointe du fruit.

KaWAI (1986c) a montré que, sur les feuilles d'aubergine, le nombre de larves est en corrélation positive avec le nombre d'adultes. Il constate aussi une corrélation positive entre les valeurs logarithmiques du nombre d'adultes et le pourcentage de fruits atteints, et une corrélation négative entre les valeurs logarithmiques du nombre d'adultes et le rendement des fruits sains. Au cours de cette étude, il a évalué le seuil de densité moyenne à 0,08 adulte par feuille sur aubergine pour que la presque totalité des fruits soient sains. Toutefois, si l'on accepte de très légères blessures sur les fruits, le seuil de nuisibilité est alors beaucoup plus élevé (largement supérieur à 20 adultes par feuille). 11 faut noter que les populations présentes dans cet essai étaient très faibles puisqu'en Guadeloupe, on a observé des populations de plus de 150 individus par feuille sur des parcelles d'aubergine non traitées (HosTACHY et al., $1986 b)$.

\section{B. Sur poivron}

Les symptômes foliaires sont identiques à eeux observés sur aubergine, et ils se manifestent sur fruits par des nécroses noires craquelées à la base, au voisinage du calice.

Sur poivron, KaWAI (1986c) a constaté qu'on ne trouve aucun individu sur feuilles lorsqu'il y a moins de 1 adulte par fleur. C'est donc le nombre d'adultes par fleur qu'il a choisi pour évaluer les dégâts. Comme sur aubergine, il constate une corrélation positive entre les valeurs logarithmiques du nombre d'adultes et le pourcentage de fruits atteints, et une corrélation négative entre les valeurs logarithmiques du nombre d'adultes et le rendement des fruits sains. Il a évalué le seuil de densité moyenne à 0,11 adulte par fleur. Là encore, l'acceptation de fruits très légèrement blessés permet de tolérer des niveaux de population largement supérieurs à 20 adultes par fleur.

\section{Sur concombre}

Les symptômes sur feuilles sont identiques à ceux observés dans le cas de l'aubergine mais ils sont visibles sur les 2 faces du feuillage (DENOYEs et al., 1986).

KAWAI (1986b) a montré sur concombre l'existence d'une corrélation positive entre le nombre de larves par feuille et le nombre d'adultes par feuille $(r=0,890$ : significatif pour un risque de 1 p. 100). D'autre part, il existe une très forte corrélation négative entre le nombre de thrips par feuille et le rendement total par plante $(r=-0,917:$ significatif pour un risque de 1 p. 100). La corrélation négative est moins forte entre le nombre de thrips par feuille et le rendement des fruits sains par plante $(r=-0,860$ : significatif pour un risque de 1 p. 100). Enfin, on observe une corrélation positive entre le nombre de thrips par plante et le pourcentage de fruits atteints par plante $(r=0,692$ : significatif pour un risque de 5 p. 100).

L'établissement de ces relations a permis d'évaluer le seuil de densité moyenne d'adultes par feuille à :

- 5,3 pour une perte de rendement total de $5 \mathrm{p} .100$ et 10,6 pour une perte de rendement total de 10 p. 100 ;

- 4,4 pour une perte de rendement des fruits sains de 5 p. 100 et 8,8 pour une perte de rendement des fruits sains de 10 p. 100.

Outre les pertes de rendement, on constate un retard de croissance des pieds atteints.

En Guadeloupe, la densité de population a dépassé dans certains cas 250 individus par feuille dans des parcelles non traitées (HostachY et al., 1986e). Ces résultats ne sont donc donnés qu'à titre indicatif et ne sont représentatifs que des conditions particulières de cette expérimentation.

KAWAI (1983a) a étudié la dynamique des populations de Thrips palmi sur concombre en serres et a constaté que le taux d'accroissement intrinsèque de la population est 0,05 par jour. C'est-à-dire que lorsque le milieu est illimité, la population est susceptible de s'accroître de 5 p. 100 par jour. Une telle situation est bien réelle lorsqu'une population de Thrips palmi s'installe sur une culture de concombre en serre; la croissance de la population est alors exponentielle. Cette croissance se poursuit de manière exponentielle jusqu'à 100 adultes par feuille, aussi bien sous serre (KAWAI, 1983a) qu'en plein champ (KAWAI, 1984).

Les adultes se trouvent surtout sur les feuilles médianes ( 5 à 10 feuilles en dessous de la feuille apicale) avant la taille et sur les feuilles apicales après la taille. Les larves se trouveront de préférence sur les feuilles inférieures (10 à 15 feuilles en dessous de la feuille apicale) c'est-à-dire à l'endroit où se trouvait la plupart des adultes 7 jours plus tôt.

La distribution des adultes dans la culture est quelconque tandis que celle des larves est modérément contagieuse. Au niveau de la feuille, la distribution des adultes et des larves est fortement contagieuse.

\section{MÉTHODES D'ÉLEVAGE DE THRIPS PALMI}

\section{A. Elevage de masse}

Au Japon Kawal (comm. pers.) réalise l'élevage de masse de Thrips palmi dans une chambre de $1 \mathrm{~m}$ de largeur, $1 \mathrm{~m}$ de profondeur et 1,20 $\mathrm{m}$ de hauteur. L'une des faces de cette chambre est constituée par une grande vitre de verre et l'élevage est réalisé en lumière naturelle, à une température comprise entre $20^{\circ} \mathrm{C}$ et $30^{\circ} \mathrm{C}$. L'humidité n'est pas contrôlée. La chambre contient 20 pieds de concombre en pots, de $1 \mathrm{~m}$ de hauteur. Tous les 3 à 4 jours, les feuilles sèches sont retirées de même que les pieds fanés qui sont remplacés par de jeunes plants; les pieds les plus récemment introduits sont placés à proximité de la vitre. Les adultes de Thrips palmi ont un phototropisme positif et s'y rendent donc d'eux-mêmes. En moyenne, un plant se dessèche au bout de 2 semaines 
du fait des attaques du thrips. Il arrive que la chambre soit envahie par des pucerons qui éliminent alors le thrips par concurrence. Dans ce cas, on effectue des traitements insecticides avec des produits à faible action sur Thrips palmi tels que le dichlorvos. Notons qu'une telle concurrence existe aussi en pots et au champ et la présence d'Aphis gossypi, entraîne là encore une forte diminution de la population de Thrips palmi et le fait disparaître à son profit (KAWAI, 1985b).

En Guadeloupe, nous avons tenté de réaliser un élevage de masse de Thrips palmi sur concombre et aubergine en serre en recouvrant le sol d'une couche de 2 à $3 \mathrm{~cm}$ de terre humidifiée fréquemment. Nous avons aussi utilisé la méthode préconisée par KAWAI. Dans les 2 cas, le développement larvaire s'est déroulé correctement et les larves de deuxième stade matures présentaient le comportement normal de nymphose décrit dans le paragraphe (V.A.). Cependant nous n'avons jamais obtenu de réinfestation à partir de ces individus, soit qu'il y ait eu une mortalité presque complète des nymphes, soit que les adultes n'aient pas trouvé les conditions nécessaires à leur développement ou à la ponte. La maitrise de ce point est indispensable pour obtenir un élevage de masse permettant d'avoir quotidiennement des individus en nombre suffisant afin d'étudier la biologie de cet insecte dans sa phase tellurique et celle de ses ennemis naturels.

Nous nous sommes aussi heurtés à la présence de pucerons appartenant aux 2 espèces Aphis gossypii et Mysus persicae qui éliminent Thrips palmi par concurrence et accroissent l'affaiblissement de la plante hôte.

\section{B. Elevage expérimental}

\section{Méthode utilisée à Taiwan par WANG \& CHU ( 1986 b)}

- Les adultes sont élevés dans un tube de verre de $4,3 \mathrm{~cm}$ de hauteur sur $1,5 \mathrm{~cm}$ de diamètre, avec au fond un papier absorbant imbibé de $0,05 \mathrm{ml}$ d'eau. Un fragment de feuille de potiron de $1,5 \mathrm{~cm}$ sur $1,5 \mathrm{~cm}$ sert de nourriture et de substrat de ponte. Il est renouvelé tous les 1 à 3 jours.

Les larves de premier stade sont élevées sur une feuille de potiron placée sur de la gaze humide.

- Les larves âgées sont élevées dans un tube identique à celui utilisé pour les adultes, avec comme site de nymphose un rouleau de papier imbibé de $0,2 \mathrm{ml}$ d'eau.

La température est de $25^{\circ} \mathrm{C}$ et la photopériode de $12 \mathrm{~h}$.

Dans ces conditions, la fécondité des femelles est de $23,0 \pm 11,6$ œufs par femelle et les dimensions de la tête, de l'ensemble du corps et des ailes ont été mesurées et ne diffèrent pas de manière significative de celles des insectes prélevés dans la nature. Les durées des différents stades de développement, les taux d'éclosion des æufs, les taux de survie et d'émergence sont les suivants :
Durée en jours Taux de survie ouf

larve de $1^{\mathrm{cr}}$ stade

larve de $2^{\circ}$ stade

nymphe

total des stades pré-imaginaux

adulte mâle

adulte femelle

$$
\begin{array}{r}
4,89 \pm 0,76 \\
1,59 \pm 0,33 \\
1,86 \pm 0,06 \\
5,39 \pm 1,03 \\
13,73 \\
22,90 \pm 5,08 \\
24,64 \pm 4,39
\end{array}
$$

\section{$98,9 \%$}

$97,2 \%$

$96,0 \%$

66,6

$61,5 \%$

\section{Méthode employée au Japon}

KAWAI (1985a) préconise l'utilisation de petites boîtes en matière plastique de $60 \mathrm{~mm}$ de diamètre et $30 \mathrm{~mm}$ de hauteur, fermées hermétiquement. Au fond de chaque boîte, on place du coton hydrophile humide dans le cas de larves ou du tissu humide dans le cas d'adultes avec pour l'alimentation un morceau de feuille de $5 \mathrm{~mm}$ sur $5 \mathrm{~mm}$. Il est indispensable de veiller à ce que l'humidité ne soit pas excessive afin que les insectes ne soient pas noyés. Lorsque les larves de deuxième stade ont atteint leur maturité, elles pénètrent entre les fibres de coton à la recherche d'un site de nymphose. Ceci évite de devoir les récolter.

Le fragment de feuille est renouvelé tous les jours. Les femelles déposent leurs œufs dans les tissus de la feuille et leur comptage est aisé à l'aide d'une loupe binoculaire de grossissement 20. Les feuilles contenant des œufs peuvent être laissées dans les boîtes jusqu'à éclosion des jeunes larves.

\section{Méthode utilisée en Guadeloupe}

En Guadeloupe, nous avons testé une méthode d'élevage expérimental qui a donné des résultats satisfaisants. Nous l'avons réalisée dans des boîtes en matière plastique à fond carré de $2 \mathrm{~cm}$ de côté sur $1 \mathrm{~cm}$ de hauteur. La nourriture apportée est un fragment de feuille de $1 \mathrm{~cm}^{2}$. L'apport de papier filtre humide n'est pas nécessaire et même parfois néfaste, le végétal apportant suffisamment d'humidité. De plus, la nymphose peut se dérouler directement sur la matière plastique de la boîte.

\section{BIOLOGIE ET DYNAMIQUE DES POPULATIONS}

\section{A. Déroulement de la vie de l'insecte}

La femelle commence à pondre le troisième jour après son éclosion (KAWAI, 1985a). Les œufs sont insérés à l'aide de la tarière sous l'épiderme du végétal. Au cours de sa vie larvaire, qui comprend 2 stades, l'insecte se nourrit du végétal non pas en suçant la sève des nervures mais en lysant le contenu des cellules du parenchyme afin de l'aspirer. La nymphose comprend 2 stades (la pronymphe et la nymphe) et se déroule dans le sol ainsi que l'a montré ETIENNE en Guadeloupe. L'adulte (fig. 3) a une vie aérienne et se nourrit de la même manière que la larve.

YoshimaRa \& KawaI (1982) ont montré que la femelle de Thrips palmi est capable de parthénogenèse arrhénotoque et KAWAI (1987a) a constaté aussi que le 


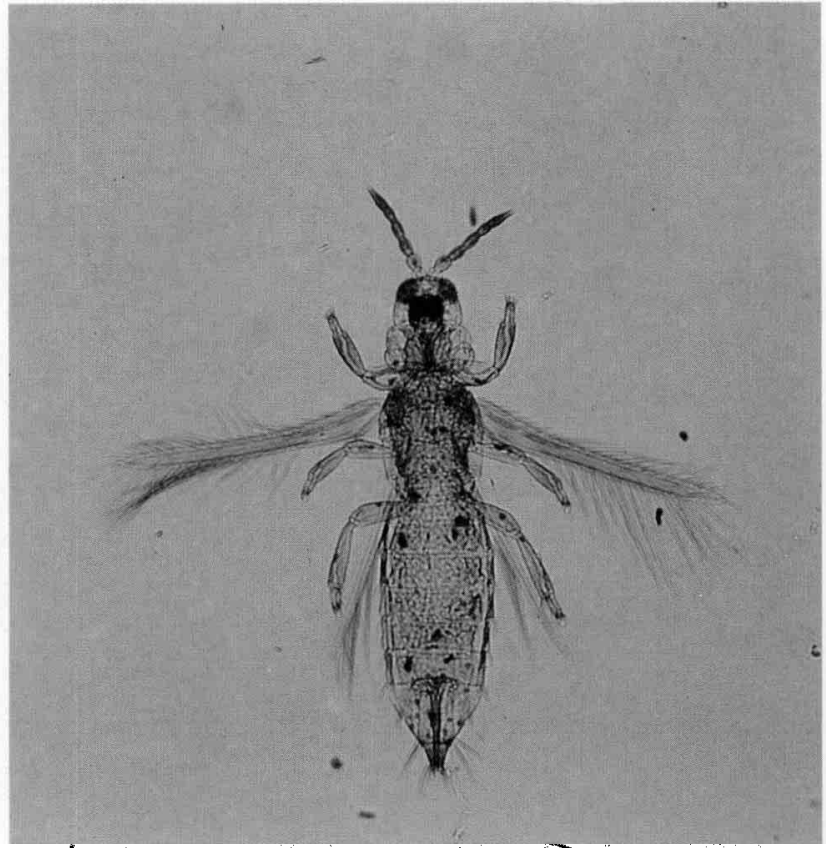

Figure 3

Fenelles de Thrips palmi.

taux de copulation dépend étroitement de la densité de population. Il est faible en cas de faible densité. Il a qualifié ce phénomène d'«effet des faibles densités ".

Une étude que nous avons réalisée en Guadeloupe dans une serre d'aubergine non traitée nous a montré que les larves de deuxième stade matures quittent le végétal pour se nymphoser non pas en suivant la tige mais en se laissant tomber sur le sol. Ce comportement a pour conséquence une répartition des nymphes sur l'ensemble de la surface cultivée et exclut les traitements localisés du sol avec un produit non systémique. Cette même étude a permis de constater que cette phase de la vie de l'insecte se déroule de préférence en milieu et en fin d'après-midi ( $14 \mathrm{~h}$ à $17 \mathrm{~h}$ ), alors que la période la plus chaude de la journée ( $11 \mathrm{~h}$ à $14 \mathrm{~h}$ ) est celle où les captures ont été les moins nombreuses. Le nombre de larves matures récoltées par heure est intermédiaire la nuit $(17 \mathrm{~h}$ à $8 \mathrm{~h})$ et dans la matinée $(8 \mathrm{~h}$ à $1 \mathrm{l} \mathrm{h})$.

\section{B. Effet de la plante hôte sur la biologie de Thrips palmi}

La biologie et la dynamique des populations de Thrips palmi ont été étudiées en laboratoire par KAWAI (1986a) sur 11 plantes hôtes ainsi qu'en présence d'eau uniquement et en diète totale. La température choisie pour cette expérimentation était de $25^{\circ} \mathrm{C}$ alors que la photopériode était de $16 \mathrm{~h}$. Les plantes testées figurent dans la liste en bas de page.

Le tableau 1 montre que les taux de survie au cours des périodes larvaire et nymphale nourries sur concombre, melon, courgette, paroka, aubergine et haricot sont élevés, ils sont moins forts sur poivron, faibles sur gombo et chrysanthème, tandis que les larves nourries sur fraisier et tomate n'atteignent pas le stade nymphal.

La durée des stades pré-imaginaux est supérieure sur gombo et chrysanthème par rapport à ce que l'on trouve sur les autres plantes.

La répartition des sexes est dans tous les cas favorable aux femelles mais il n'est pas possible de tirer plus de conclusions des informations apportées dans cette publication.

La longévité des adultes nourris sur concombre, courgette, aubergine et haricot est plus élevée que sur les autres plantes hôtes et là encore les chiffres obtenus sur fraisier, tomate et chrysanthème sont très faibles.

La fécondité des femelles est élevée sur concombre et largement supérieure à celle obtenue avec les autres plantes hôtes.

Le tableau 2 permet de constater que la durée de génération n'est pas différente entre les plantes hôtes.

En revanche, l'accroissement de la population est maximal sur concombre (Ro, r, Rm), plus faible mais toujours élevé sur melon et aubergine. Parmi toutes celles étudiées au Japon, ces 3 plantes sont de loin les plus favorables à l'insecte.

\section{Effet de la température sur la biologie de Thrips palmi}

On doit à KAWAI (1985a) cette expérimentation effectuée en laboratoire, sur concombre, avec une photopériode de $16 \mathrm{~h}$ qui, il faut le noter, n'existe pas dans les Antilles.

Le développement de Thrips palmi nécessite une température minimale de $11,6^{\circ} \mathrm{C}$ alors que l'accomplissement des stades pré-imaginaux requiert une somme de températures journalières de $189,1^{\circ} \mathrm{C}$.

On constate que le déroulement du cycle de Thrips palmi est le plus rapide à $30^{\circ} \mathrm{C}$ mais la fécondité des femelles et la vitesse de ponte sont maximales à $25^{\circ} \mathrm{C}$. A cette température, une femelle peut pondre jusqu’à

$\begin{array}{ll}\text { Nom anglais } & \text { Nom français } \\ \text { cucumber } & \text { concombre } \\ \text { melon } & \text { melon } \\ \text { pumpkin } & \text { courgette } \\ \text { balsam pear } & \text { paroka } \\ \text { eggplant } & \text { aubergine } \\ \text { sweet pepper } & \text { poivron } \\ \text { lomato } & \text { tomate } \\ \text { kidney bean } & \text { haricot } \\ \text { okra } & \text { gombo } \\ \text { strawberry } & \text { fraise } \\ \text { chrysanthemum } & \text { chrysanthèmc }\end{array}$

Sachimidori

Earl's favourite Ebisu

Shin-nagasakinaga New acc

Toko-K

America

Harunok: Shun-no-chikara 


\section{TABLEAU}

Principales données de la biologie de Thrips palmi sur differentes plantes hôtes (d'après KaWl. 1986a).

Biology' of Thrips palmi on various crops.

\begin{tabular}{|c|c|c|c|c|c|c|c|c|c|}
\hline & $\begin{array}{l}(1) \\
\% \\
\text { PN }\end{array}$ & $\begin{array}{l}(2) \\
\% \\
\mathrm{~N}\end{array}$ & $\begin{array}{l}(3) \\
\% \\
\text { A }\end{array}$ & $\begin{array}{c}(4) \\
\% \\
F\end{array}$ & $\begin{array}{c}(5) \\
\text { durée } \\
\text { st. larv. }\end{array}$ & $\begin{array}{c}(6) \\
\text { durée } \\
\text { st. pr.imp. }\end{array}$ & $\begin{array}{l}\text { (7) } \\
\text { longévité } \\
\text { femelles }\end{array}$ & $\begin{array}{l}\text { (8) } \\
\text { fécondité } \\
\text { femelles }\end{array}$ & $\begin{array}{c}(9) \\
\text { vitesse } \\
\text { de ponte }\end{array}$ \\
\hline concombre & 74 & 72 & 66 & 71,2 & $5.1 \pm 0.2$ & $9,2 \pm 0,2$ & $15,8 \pm 3,6$ & $59,6 \pm 8,5$ & $3,8 \pm 0,6$ \\
\hline melon & 70 & 55 & 50 & 78,8 & $4.7 \pm 0.2$ & $9,0 \pm 0,3$ & $13,3 \pm 2,8$ & $32,5 \pm 13,1$ & $2,4 \pm 0,4$ \\
\hline courgette & 70 & 53 & 43 & 79,1 & $3.9 \pm 0.2$ & $8.3 \pm 0.3$ & $15,8 \pm 2,4$ & $21,5 \pm 7,2$ & $1,4 \pm 0,3$ \\
\hline paroka & 77 & 65 & 61 & 57,3 & $5.0 \pm 0.3$ & $9.4 \pm 0,2$ & $14,4 \pm 2,9$ & $7.8 \pm 1.7$ & $0,5 \pm 0,1$ \\
\hline aubergine & 74 & 71 & 62 & 85,5 & $5.0 \pm 0.2$ & $9,1 \pm 0,2$ & $15,5 \pm 2,9$ & $25,1 \pm 4.4$ & $1,6 \pm 0,4$ \\
\hline poivron & 53 & 50 & 45 & 71,1 & $4.7 \pm 0.4$ & $9,0 \pm 0,4$ & $11,8 \pm 2,9$ & $9,0 \pm 3,2$ & $0,8 \pm 0,2$ \\
\hline tomate & 0 & 0 & 0 & - & & - & $5,1 \pm 0,8$ & $0^{-}$ & 0 \\
\hline haricot & 69 & 69 & 65 & 73,8 & $5.1 \pm 0.2$ & $9.2 \pm 0.2$ & $15,0 \pm 3,6$ & $9,8 \pm 3,0$ & $0,7 \pm 0,3$ \\
\hline gombo & 23 & 23 & 20 & 90,0 & $7.2 \pm 0.5$ & $11.7 \pm 0.6$ & $12.4 \pm 2.1$ & $2.8 \pm 0,9$ & $0,2 \pm 0,1$ \\
\hline fraise & 0 & 0 & 0 & - & - & - & $4,0 \pm 0,3$ & $0^{-}$ & $0^{-}$ \\
\hline chrysanthème & 20 & 19 & 13 & 84,6 & $6,5 \pm 0,7$ & $11,2 \pm 1,2$ & $6,1 \pm 0.4$ & $0,6 \pm 0.4$ & $0,1 \pm 0,1$ \\
\hline eau & -- & $-\cdots$ & - & - & - & - & $3,9 \pm 0,2$ & 0 & 0 \\
\hline diète totale & - & - & - & 一 & - & - & $2,0 \pm \quad 0,0$ & 0 & 0 \\
\hline
\end{tabular}

(1) : pourcentage de prénymphoses (par rapport au nombre d'oufs pondus).

(2) : pourcentage de nymphoses (par rapport au nombre d'oeufs pondus).

(3) : pourcentage d’émergences d'adultes (par rapport au nombre d'œufs pondus).

(4) : pourcentage de femelles dans la population.

(5): durée de la phase larvaire (en jours).

(6) : durée des stades pré-imaginaux (en jours).

(7) : longévité des femelles (en jours).

(8): fécondité des femelles (nombre d'œufs pondus au cours de la vie d'une femelle).

(9) : vitesse de ponte (en œufs pondus par femelle et par jour).

TABLEAU 2

Quelques données de la dynamique des populations de Thrips palmi sur différentes plantes hötes (d'apres KaWA, 1986a).

Population grenth of Thrips palmi on various crops.

\begin{tabular}{|c|c|c|c|c|}
\hline & $\begin{array}{l}\text { Duréc de } \\
\text { génération } \\
\text { (en jours) }\end{array}$ & $\begin{array}{l}\text { Tatux net de } \\
\text { reproduction } \\
\text { Ro }_{0}\end{array}$ & $\begin{array}{c}\text { Taux } \\
\text { d'accroisscment } \\
\text { naturel/jour (r) }\end{array}$ & $\begin{array}{c}\text { Taux de } \\
\text { reproduction } \\
\text { par mois }(\mathrm{Rm})\end{array}$ \\
\hline concombre & 24,8 & 28,0 & 0.134 & 55,7 \\
\hline melon & 23,2 & 13,0 & 0,111 & 27.9 \\
\hline courgette & 24,8 & 7,3 & 0,080 & 11.0 \\
\hline paroka & 25,0 & 2.7 & 0,040 & 3,3 \\
\hline aubergine & 25,4 & 13.3 & 0,102 & 21.3 \\
\hline poivron & 22,8 & 2.9 & 0,047 & 4.1 \\
\hline haricot & 25,2 & 4,7 & 0.061 & 6.2 \\
\hline gombo & 25,9 & 0,5 & 0,027 & 0.4 \\
\hline chrysanthème & 21,2 & 0,1 & -0.109 & 0.0 \\
\hline
\end{tabular}

60 œufs en 16 jours environ (tabl. 3 ). Le taux de reproduction est maximal à $25^{\circ} \mathrm{C}$ tandis que le taux intrinsèque d'accroissement naturel par jour et le taux de reproduction par mois atteignent leurs maxima à $30^{\circ} \mathrm{C}$. A $25^{\circ} \mathrm{C}$, le taux intrinsèque d'accroissement naturel par jour est de 0,134 , c'est-à-dire que la population peut s'accroître de 13,4 p. 100 par jour si les ressources du milieu ne sont pas limitantes; à cette même température, le taux de reproduction par mois est de 55,7.

\section{LUTTE}

\section{A. Lutte biologique et variétés résistantes}

Jusqu'à présent, aucune source de résistance n'a été détectée et aucun pathogène ou parasite n'a été décrit.
Cependant, MEssiaen (comm. pers.) et nous même avons observé 2 individus de Thrips palmi attaqués par un champignon pathogène. Cependant, ceci représente une très faible proportion sur les quelques 70000 thrips que nous avons récoltés depuis la mise en place des essais actuellement en cours à Saint-François en Guadeloupe. Toutefois, il semblerait que les attaques de thrips soient moins fortes dans les zones les plus humides de l'île volcanique de Basse-Terre et il n'est donc pas à exclure un rôle important des pathogènes dans certaines conditions de milieu, en particulier climatiques.

Les principaux prédateurs cités dans le monde sont des Hétéroptères Anthocoridés du genre Orius et des Acariens de la famille des Phytoseiidae :

Orius similis Zheng en Chine où WEI et al. (1984) ont $\mathrm{pu}$ constater au laboratoire qu'un seul individu est 
TABLEAU 3

Principales données de la biologie et de la dynamique des populations de Thrips palmi à 4 températures (d'après KaWAl, 1985a).

Biology and population growth of Thrips palmi at different temperatures.

\begin{tabular}{|c|c|c|c|c|}
\hline & $15^{\circ} \mathrm{C}$ & $20^{\circ} \mathrm{C}$ & $25^{\circ} \mathrm{C}$ & $30{ }^{\circ} \mathrm{C}$ \\
\hline Durée du stade cuf (1) & $18,3 \pm 2,0$ & $10,4 \pm 1,6$ & $6,3 \pm 0,7$ & $5,1 \pm 0,3$ \\
\hline Durée de la phase larvaire (1) & $21,7 \pm 3,0$ & $7,8 \pm 1,1$ & $4,8 \pm 0,8$ & $4,8 \pm 0,8$ \\
\hline Durée de la nymphose (1) & $13,7 \pm 0,7$ & $6,6 \pm 0,8$ & $3,5 \pm 0,5$ & $3,0 \pm 0,2$ \\
\hline Durée totale des stades pré-imaginaux (1) & 53,7 & 23,8 & 13,6 & 11,9 \\
\hline Longévité des femelles (1) & $35,9 \pm 11,4$ & $22,8 \pm 10,4$ & $15,8 \pm 9,2$ & $10,9 \pm 7,1$ \\
\hline Longévité des mâles (1) & $35,7 \pm 13,2$ & $20,6 \pm 13,6$ & $13,2 \pm 11,0$ & $11,3 \pm 8,3$ \\
\hline Pourcentage d'éclosion des œufs (2) & 92 & 97 & 98 & 96 \\
\hline Pourcentage de nymphoses (2) & 65 & 70 & 74 & 73 \\
\hline Pourcentage d'éclosion d'adultes (2) & 62 & 65 & 66 & 68 \\
\hline Pourcentage de femelles (2) & 82,3 & 81,5 & 71,2 & 79,4 \\
\hline Fécondité (3) & $32,4 \pm 19,1$ & $48,8 \pm 23,2$ & $59,6 \pm 20,7$ & $35,4 \pm 31,2$ \\
\hline Vitesse de ponte (4) & $0.9 \pm 0,4$ & $2,1 \pm 0,4$ & $3,8 \pm 1,4$ & $3,3 \pm 2,2$ \\
\hline Durée de génération (d'œuf à œuf) (1) & 80,2 & 40,7 & 24,8 & 20,5 \\
\hline Taux de reproduction (Ro) & 16,5 & 25,9 & 28,0 & 19,1 \\
\hline Taux d'accroissement naturel par jour (r) & 0,035 & 0,080 & 0,134 & 0,144 \\
\hline Taux de reproduction par mois $(\mathrm{Rm})$ & 2,9 & 11,0 & 55,7 & 75,2 \\
\hline
\end{tabular}

(1): en jours

(2): pourcentages par rapport au nombre d'œufs pondus

(3) : œufs par femelle

(4) : œufs par femelle et par jour

Ro: nombre de descendants adultes qu'est susceptible d'avoir une femelle.

$r$ : facteur d'accroissement de la population lorsque le milieu est illimité.

$\mathrm{Rm}$ : nombre de descendants adultes qu'est susceptible d'avoir une femelle en 1 mois.

capable de consommer près de 440 individus de Thrips palmi au cours de sa vie. Dans la région de Guangzhou, $O$. similis présente 14 générations annuelles, chacune d'elles durant de 17 à 64 jours environ selon la température.

Orius maxidentex Ghauri est présent en Inde (KUMAR \& ANANTHAKRISHNAN, 1984) où il est un important prédateur de Thrips palmi sur jeunes feuilles de sésame, puis après la récolte sur une mauvaise herbe, Croton sparsiflorus.

Le genre Orius est aussi présent à Hawaï (JoHnson, 1986) où il est le seul prédateur de Thrips palmi recensé à ce jour. Cependant, il peut présenter des niveaux de population assez élevés ( 0,8 individu par feuille).

Le genre Orius existe également au Japon sur les concombres infestés par Thrips palmi. Des essais menés en laboratoire par KAJITA (1986) sur des fragments de jeunes feuilles ou de feuilles médianes de concombre de $2 \mathrm{~cm}^{2}$ ont montré que ces punaises à leur deuxième stade larvaire préfèrent consommer les larves (de premier et de deuxième stade indistinctement) mais sont aussi capables de se nourrir d'adultes en l'absence de larves. Une punaise du genre Orius peut consommer 1,4 larve de premier stade en $24 \mathrm{~h}$ lorsque l'expérience est réalisée sur une jeune feuille et 4,1 lorsqu'on utilise une feuille médiane. Il apparaît ici un effet significatif de l'âge de la feuille sur la consommation de larves de premier stade.
Au Japon, KaJiTA (1986) a aussi observé des acariens de la famille des Phytoseiidae prédateurs de Thrips palmi sur concombre. Ils appartiennent à 2 espèces différentes : Amblyseius mckenziei Schuster et Pritchard et Amblyseius okinawanus Ehara. Les femelles de ces 2 espèces préfèrent consommer des larves de premier stade plutôt que des larves de deuxième stade ou que des adultes. Ainsi, sur une jeune feuille de concombre, la consommation en larves de premier stade sur une période de $24 \mathrm{~h}$ est de 0,2 pour A.mckenziei et de 1,0 pour A. okinawanus. Ces chiffres ne diffèrent pas significativement de ceux obtenus pour Orius.

\section{B. Lutte chimique}

Plusieurs matières actives ont été testées en particulier au Japon. Nous citerons ici celles qui ont donné les résultats les plus encourageants. Précisons que certaines d'entre elles ne sont pas homologuées en France (prothiofos, mecarbam) ou ne le sont que sous forme granulée (carbosulfan).

- le carbosulfan en concentré émulsifiable à la dose de $0,25 \mathrm{~kg} \mathrm{~m}$.a. par hectare s'est avéré le plus efficace des produits testés au Japon sur pomme de terre, aussi bien sur larves que sur adultes (ORITA et al., 1982). Le carbosulfan peut aussi être utilisé en granulés à la plantation en traitement du sol localisé à raison de $2,0 \mathrm{~g}$ 
de matière active par plant. Il est alors très efficace et son action se prolonge pendant plusieurs mois (KAWAI, 1986c).

- l'oxamyl a été utilisé à Hawaï à la dose de $1,2 \mathrm{~kg}$ m.a. par hectare (JOHNSON, 1986). On a constaté qu'il réduit la densité de larves mais ne semble pas avoir d'action sur les adultes. Au Japon, la dose utilisée était de $1,5 \mathrm{~kg} \mathrm{~m}$.a. par hectare sous forme granulée (ORITA et al., 1982).

- le prothiophos en concentré émulsifiable à $0,45 \mathrm{~kg}$ m.a. par hectare s'est avéré efficace sur pomme de terre au Japon (ORITA et al., 1982).

- le chlorfenvinphos (concentré émulsifiable) à $0,48 \mathrm{~kg} \mathrm{~m} . \mathrm{a}$. par hectare additionné de mancozèbe en poudre mouillable $(1,5 \mathrm{~kg} \mathrm{~m}$.a. par hectare) utilisé au Japon sur pomme de terre a donné des résultats moyens (ORITA et al., 1982).

- le mecarbam (concentré émulsifiable) à $0,36 \mathrm{~kg}$ m.a. par hectare a été moyennement efficace sur pomme de terre au Japon (ORITA et al., 1982).

- le profenofos est conseillé à des doses de 0,5 à $0,75 \mathrm{~kg} \mathrm{~m} . \mathrm{a}$. par hectare environ tous les 7 jours sur aubergines et sur Cucurbitacées en Guadeloupe et Martinique. Cependant, ce produit ne peut pas ètre utilisé moins de 40 jours avant la récolte et son efficacité ne dure que 2 ou 3 semaines après son application; les essais du GRISP Antilles-Guyane n'ont pas mis en évidence un produit efficace applicable pendant la période de floraison (Hostachy et al., 1986a, b, c, d, e).

La biologie de Thrips palmi est particulièrement importante dans la définition du programme de traitement.

Ainsi, du fait de l'existence de l'effet des faibles densités de population que nous avons déjà cité, KAwAI (1987b) constate que la fréquence des traitements foliaires nécessaires décroit lorsque l'on fixe un seuil d'intervention de plus en plus faible. A partir de cette observation, il estime préférable de déterminer ce seuil aussi bas que possible, en tenant compte du fait que plus on considère de faibles densités de population, plus leur suivi est difficile et nécessite l'observation d'un nombre croissant de plants. Les interventions doivent donc, selon lui, débuter le plus tôt possible après le début de l'infestation.

D'autre part, le stade œuf et le stade nymphal étant pratiquement épargnés par les traitements foliaires, KAWAI (1987b) préconise un premier traitement lorsque le seuil d'intervention est atteint, suivi quelques jours plus tard d'une deuxième application quelle que soit la densité de population. Ainsi, en choisissant correctement cet intervalle, les individus qui n'ont pas été touchés lors du premier traitement sont détruits lors de la deuxième pulvérisation. Cette méthode permet de réduire la fréquence des traitements par rapport à celle qui consiste en une intervention à chaque dépassement du seuil.

La méthode des traitements périodiques sans considération du niveau de population présente une efficacité certaine mais nécessite une fréquence légèrement supérieure à la technique précédente. En revanche, elle évite le suivi permanent des niveaux de population.

Ces résultats, obtenus par un modèle de simulation sur concombre en serre, confirment un certain nombre de travaux que nous citerons ici à titre d'exemples: MATSUZAKI (1983) constate que sur aubergine, les insec- ticides doivent être appliquées 2 ou 3 fois à 4 ou 5 jours d'intervalles à partir du début de l'infestation; NAGAI \& NONAKA (1983), estiment sur poivron que les pulvérisations doivent intervenir 2 ou 3 fois à 5 jours d'intervalles dans les cas de fortes densités alors qu'elles peuvent être espacées de 15 jours lorsqu'elles sont effectuées sur de très faibles densités de populations.

\section{Lutte agronomique}

Plusieurs méthodes ont été testées mais leur efficacité reste moyenne.

- la couverture du sol par un film plastique transparent réduit fortement la densité de populations et reste efficace longtemps après le retrait du film (NASU $e t$ al., 1986).

- en serre, la couverture du sol par un film de polyéthylène argenté réduit la densité de population de plus de 90 p. 100 selon MAKINO (1984) ou de 80 à 90 p. 100 selon NAGAI (1981) par rapport à une culture sur sol nu et de 67 p. 100 par rapport à une culture sur sol recouvert de polyéthylène noir (Suzukı \& MiYara, 1984).

- la couverture des serres en plastique par un film de vinyl absorbant les ultraviolets a été utilisée avec succès sous serres de melon et comparée à un témoin de plastique normal : la dispersion et le vol des adultes sont plus limités dans le cas du plastique absorbant dans les ultraviolets; de même, l'immigration est plus faible et l'émigration supérieure à ce qu'on observe avec le plastique standard (KAWAI, 1986d). NonAKa \& NAGAI (1983) estiment que l'immigration est alors réduite de 90 p. 100.

la couverture des côtés ouverts de la serre par une maille fine est efficace mais les fruits obtenus sont de qualité inférieure en raison de l'augmentation de température (environ $5^{\circ} \mathrm{C}$ ) que cela entraîne (NASU et al., 1986).

- la couverture complète des plants d'aubergine par une maille fine a donné de bons résultats sur les populations de faible densité mais il en résulte une mauvaise coloration des pieds d'aubergine (NAKAMURA et al., 1984). Sur concombre, cette méthode permet d'avoir des densités de population de 75 à 90 p. 100 selon NAGai (1981) et de 80 à 98 p. 100 selon Suzuki \& MiYara (1984) inférieures à celles observées en l'absence de protection.

- des méthodes telles que la pulvérisation d'insecticides sur des semis avant la plantation, le traitement ou l'arrachage des mauvaises herbes à proximité des semis sont efficaces mais uniquement s'il n'y a pas de réinfestation venue de l'extérieur.

- aux Philippines lors de la mise en place de cultures de niébé après du riz irrigué, l'absence de labour et le maintien des chaumes de riz réduisent nettement l'effet de Thrips palmi pendant environ 2 semaines. Le paillage a le même effet (RUHENDi \& Litsinger, 1979 ; LitsinGER \& RUHENDI, 1984).

\section{Lutte par piégeage}

Les adultes de Thrips palmi sont attirés par le bleu et le blanc (YAmAmoto et al., 1981; Kitakata \& 
YoshIDA 1982). Ainsi, au Japon, l'utilisation de rubans bleus englués placés tous les 2 à $3 \mathrm{~m}^{2}$ donne de bons résultats en cultures maraîchères sous serres (KAWAI, 1982, $1983 b$; TAKEUCHI et $a l ., 1983$; NonAKA \& NAGAI, 1984). D’après les travaux de NisHino \& ONO (1984), ce systeme permet de capturer de 3 à 9 p. 100 des adultes quand les pièges sont placés tous les $2 \mathrm{~m}^{2}$ et 1 à 3 p. 100 des adultes quand ils sont tous les $3,3 \mathrm{~m}^{2}$.

Cependant, les méthodes de piégeage ne sont efficaces que lorsqu'elles sont mises en cuvre alors que les populations de thrips sont encore très faibles. Si cette condition est respectée, les résultats peuvent être particulièrement intéressants comme le prouve le tableau 4 .

\section{E. Elimination des thrips sur les fruits récoltés}

En Guadeloupe, JACQUA et ETIENNE (1988) ont constaté que les thrips dissimulés sous le calice des fruits d'aubergine récoltés survivent à 10 jours de transport à $10{ }^{\circ} \mathrm{C}$ jusqu'en métropole, mais qu'il est possible de les éliminer complètement, par trempage des fruits récoltés dans de l'eau à $45^{\circ} \mathrm{C}$ pendant $7 \mathrm{~min}$.

\section{CONCLUSION}

Les résultats publiés au Japon donnent des informations précises sur la biologie de Thrips palmi. Aussi, les travaux menés par l'INRA en liaison avec le CIRAD en Martinique ont pour objectif la mise au point d'une méthode de lutte intégrée applicable dans les Antilles. Actuellement, les études sont menées essentiellement sur aubergine en Guadeloupe et sur concombre en Martinique.

Au niveau de l'IRAT en Martinique : des essais dans le domaine de la lutte chimique et de la lutte par méthodes culturales, et la détermination d'un seuil de nuisibilité sont en cours.

Au niveau de l'INRA Antilles-Guyane:

- la Station d'Amélioration des plantes s'occupe de la recherche de sources de résistance dans le genre Solanum aussi bien chez des espèces sauvages que chez des variétés cultivées.
- la Station de Zoologie et de Lutte Biologique mène les études sur la biologie et l'écologie de Thrips palmi. En particulier, elle cherche à réaliser un inventaire aussi large que possible des parasites et prédateurs et estimer l'impact de ces auxiliaires naturels.

Des observations réalisées en 1987 ont montré que des champs régulièrement traités au profenofos n'étaient plus convenablement protégés contre ce ravageur alors que des parcelles laissées à l'abandon du fait de la gravité des dégâts se trouvaient beaucoup moins fortement attaquées.

Ces remarques nous ont amené à orienter notre programme en priorité vers la recherche des facteurs de limitation naturelle des populations de Thrips palmi et en premier lieu les facteurs biotiques agissant au niveau du sol sur le stade nymphal et au niveau des feuilles sur les adultes et les larves. Ces essais sont en cours mais d'ores et déjà ils semblent confirmer les observations précédentes et montrer les limites de la lutte chimique et l'installation progressive d'un équilibre entre Thrips palmi et ses ennemis naturels en Guadeloupe. Les facteurs climatiques, en particulier les précipitations, paraissent aussi jouer sur ce ravageur un rôle négatif qui est loin d'être négligeable.

La mise au point d'une méthode fiable de lutte intégrée est un travail de longue haleine qui reste toutefois la seule voie raisonnable pour résoudre durablement ce problème qu'il n'est d'ailleurs pas totalement exclu de voir apparaître en régions tempérées, notamment en France métropolitaine.

Pour cela, les nombreux travaux effectués au Japon constituent pour nous une base précieuse et le renforcement des relations avec ces chercheurs est essentiel. Cependant, les techniques de lutte proposées au Japon ne sont pas directement transposables aux Antilles Françaises en raison:

- des différences dans les conditions du milieu,

- des différences dans les variétés cultivées,

- de la réglementation française plus sévère en matière de lutte chimique,

- du fait que les méthodes de piégeage et les méthodes agronomiques semblent donner d'excellents résultats sous serres mais sont difficilement utilisables en plein champ et leur succès moins assuré.

Reçu le 2 octobre 1987 Accepté le 26 avril 1988

TABLEAU 4

Efficacité du système de piégeage sur différentes cultures.

Efficiency of sticky traps on various crops.

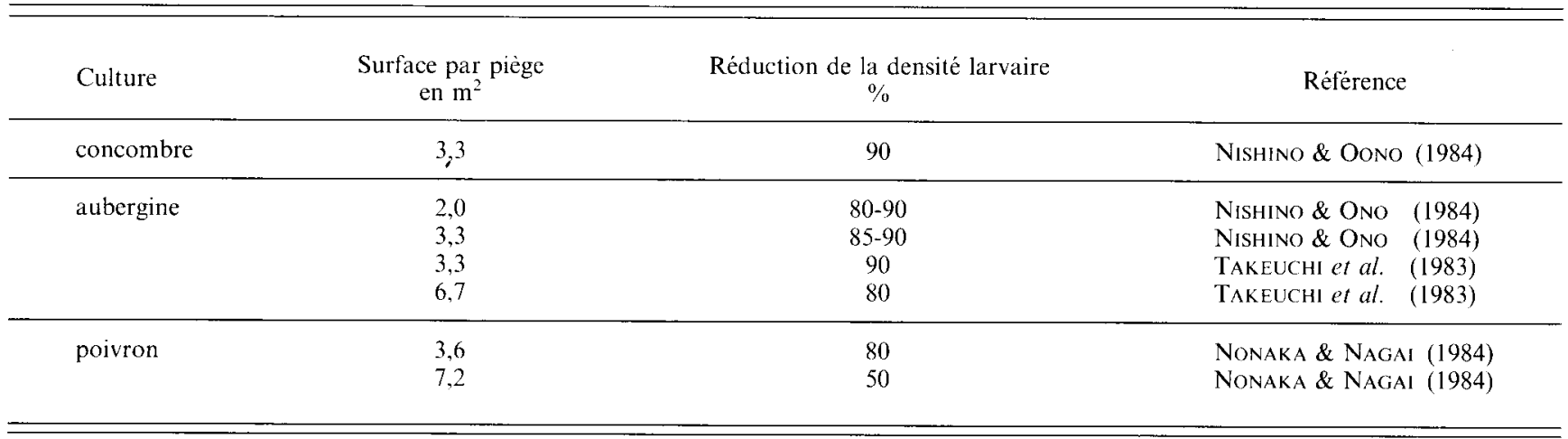




\section{RÉFÉRENCES BIBLIOGRAPHIQUES}

Bhatti J. S., 1980. Species of the genus Thrips from India (Thysanoptera). Syst. Entomol., 5, 109-166.

Bournier J. P., 1983. Un insecte polyphage: Thrips palmi (Karny) important ravageur du cotonnier aux Philippines. Cot. Fib. Trop., 38 (3), 286-288.

Bournier J. P., 1986. Sur la distribution géographique de Thrips palmi Karny. Cot. Fib. Trop., 41 (1), 59-60.

Crowe T. J., 1985. Field crop pests in Burma : an annotated list. Food and Agricultural Organization Office, Rangoon, Burma. 65 pp.

Denoyes B., Bordat D., De Bon H., Daly P., 1986. Thrips palmi Karny, a new pest of vegetable crops in Martinique West Indies., Agron Trop., 41 (2), 167-169.

Hostachy B., Jacqua G., Etienne J., Ano G., 1986a. Essais d'efficacité de quelques insecticides foliaires contre Thrips palmi sur aubergine en Guadeloupe. Document GRISP Antilles-Guyane 86/02.

Hostachy B., Jacqua G., Etienne J., Ano G., 1986b. Lutte chimique contre Thrips palmi sur aubergine en Guadeloupe. Essai d'associatinns insecticides foliaires-insecticides du sol. Document GRISP AntillesGuyane 86/03.

Hostachy B., Jacqua G., Etienne J., Ano G., 1986c. Lutte chimique contre Thrips palmi en Guadeloupe. Essai insecticide efficacité en période de récolte. Document GRISP Antilles-Guvane 86/04.

Hostachy B., Onillon H., Jacqua G., Etienne J., Ano G., 1986d. Essai d'efficacité de deux insecticides foliaires contre Thrips palmi sur concombre en Guadeloupe. Document Grisp Antilles-Guyane 86/05.

Hostachy B., Onillon H., Jacqua G., Etienne J., Ano G., 1986e. Thrips palmi sur aubergine. Testage d'un programme théorique. Document GRSIP Antilles-Guyane 86/06.

Jacqua G., Etienne J., 1988. Possibilités d'élimination du Thrips palmi sur des fruits d'aubergine par trempage dans de l'eau chaude. Bull. Agron. Antilles-Guyane, 6, 2-3.

Johnson M. W., 1986. Population trends of a newly introduced species, Thrips palmi (Thysanoptera: Thripidac), on commercial watermelon in Hawaii. J. econ. Entomol, 79 (3), 718-720.

Kajita H., 1986. Predation by Amblyseius spp. (Acarina : Phytoseiidae) and Orius sp. (Hemiptera : Anthocoridae) on Thrips palmi Karny (Thysanoptera : Thripidae). Appl. Entomol. Zool., 21 (3), 482-484.

Kawai A., 1982. Test for attractiveness of white sticky trap for Thrips palmi Karny in Japan. Proc. Assoc. Plant Prot. Kyushu, 28, 176-177.

Kawai A., 1983a. Studies on population ecology of Thrips palmi Karny. 1. Population growth and distribution on cucumber in greenhouse. Jpn. J. appl. Entomol. and Zool., 27 (4), 261-264.

Kawai A., 1983b. Studies on population ecology of Thrips palmi Karny. 4. Relationship between the density of adults on plants and the number of individuals trapped by sticky traps. Proc. Assoc. Piant Prot. Kvushu, 29, 87-89.

Kawai A., 1984. Studies on population ecology of Thrips palmi Karny 5. Population growth on cucumber in open field. Kvushu Agric. Res., 46, $121-122$

Kawai A., 1985a. Studies on population ecology of Thrips palmi Karny. 7. Effect of temperature on population growth. Jpn. J. $a_{p} p p l$. Entomol. and Zool., 29 (2), 140-143.

Kawai A., 1985b. Studies on population ecology of Thrips palmi Karny. 9. Interspecific competition with Aphis gossypii Glover. Proc. Assoc. Plant Prot. Kyushu, 31, 156-159.

Kawai A., 1986a. Studies on population ecology of Thrips palmi Karny. 10. Differences in population growth on various crops. Jpn.J. appl. Entomol. and Zool, 30 (1), 7-11.

Kawai A., 1986h. Studies on population ecology of Thrips palmi Karny. 11. Analysis of damage to cucumber. Jpn. J. appl. Entomol. and Zool., 30 (1), 12-16.

Kawai A., 1986c. Studies on population ecology of Thrips palmi Karny. 12. Analyses of damage to eggplant and sweet pepper. Jpr. J. appl. Entomol. and Zool., 30 (3), 179-187.

Kawai A., 1986d. Studies on population ecology of Thrips palmi Karny. 13. Comparison of the adult movement in a plastic greenhouse depending on the covering materials. Proc. Assoc. Plant Prot. Kyushu, $32,163-165$
Kawai A., 1987a. Studies on the population ecology of Thrips paimi Karny. 14. Relationship between the density and the rate of copulation. Jpn. J. Appl. Entomol. Zool., 31, 85-87.

Kawai A., 1987b. Studies on the population ecology of Thrips palmi Karny. 15. Evaluation of effectiveness of control methods using a simulation model. Appl. Entomol. Zool., 22 (3), 292-302.

Kitakata S., Yoshida M., 1982. Control of insects in greenhouses by colored ribbon traps. Shokubutu Boueki, 36, 478-481.

Kumar N. S., Ananthakrishnan T. N., 1984. Predator-thrips interactions with reference to Orius maxidentex Ghauri and Carayonocoris indicus Muraleedharan (Anthocoridae: Heteroptera). Proc. Indian Natl. Sci. Acad., B50 (2), 139-145.

Litsinger J. A., Ruhendi, 1984. Rice Stubble and Straw Mulch Suppression of Preflowering Insect Pests of Cowpeas Sown After Puddled Rice. Environ. Entomol., 13 (2), 509-514.

Makino S., 1984. Control of Thrips palmi by the mulching with polyethylene film. Kyushu Agric. Res., 46, 126.

Matsuzaki T., 1983. Ecology and control of Thrips palmi Karny on the eggplant in Kochi Prefecture. Shokubutu Boueki, 37, 281-283.

Nagai K., 1981. Control of Thrips palmi Karny. In Proceedings of a Symposium on the control of pest and Disease of vegetables, Japan Plant Protection Association, Tokyo, 35-43.

Nagai K., Nonaka K., 1983. Ecology and control of Thrips palmi Karny on the green pepper in Miyazaki Prefecture. Shokubutu Boueki, 37, 284-286.

Nakamura T., Tanaka Y., Ikeda H., 1984. Studies on the control of Thrips palmi Karny on eggplant, cucumber and chrysanthemum by cultural and chemical methods. Bull. Fukuoka Agric. Res. Cent., 83, $77-84$.

Nasu Y., Kimura Y., Tsuji H., 1986. Control of Thrips palmi Karny using agricultural covering materials. Proc. Kansai Plant Prot. Soc., 28, 25-29.

Nishino T., Ono K., 1984. Control of Thrips palmi Karny by sticky ribbon traps (Seiryu). Kyushu Agric. Res., 46, 124

Nonaka K., Nagai T., 1983. Ecology and control of thrips infesting fruit vegetables. 7. Control of Thrips palmi using U.V.-cut film. Kyushu Agric. Res., 45, 110-120.

Nonaka K., Nagai T., 1984. Ecology and control of thrips infesting fruit vegetables. 8. Control of Thrips palmi using blue colored sticky ribbons. Kyushu Agric. Res., 46, 125.

Orita S., Hirotani H., Nakasuka, 1982. Field evaluation of several chemicals for the control of Thrips palmi Karny on potato plant. Proc. Assoc. Plant Prot. Kyushu, 28, 142-144.

Ruhendi, Litsinger J. A., 1979. Insect-suppressing effect of rice stubble height, tillage practices, and straw mulch in a wetland rice-cowpea cropping pattern. Int. Rice Res. Newsl., 4 (3), 26-27.

Suzuki H., Miyara A., 1984. Studies on ecology and control of the southern yellow thrips, Thrips palmi Karny (Thysanoptera: Thripidac). 1. Physical control using agricultural covering materials. Bull. Okinawa Agric. Exp. Sta., 9, 85-93.

Takeuchi H., Kobayashi Y., Kitakaka S., Matsuo K., Yoshira M., Shirai E., 1983. Suppression effect of mass trapping by a sticky ribbon "Seiryu" to Thrips palmi Karny on the eggplant in a plastic greenhouse. Proc. Kanto-Tosan Plant Prot. Soc., 30, 146-147.

Wang C.-L., Chu Y.-I., 1986a. Review of the southern yellow thrips, Thrips palmi Karny. Chin. J. Entomol., 6, 133-143.

Wang C.-L., Chu Y.-I., 1986b. Rearing method of the southern yellow thrips, Thrips palmi Karny, in Laboratory. Plant Prot. Bull. (Taiwan, R.O.C.), 28, 407-411.

Wang C.-L., Chu Y.-I., 1987. The identification of thrips on cucurbits J. Agric. Res. China, 36 (4), 429-434.

Wangboonkong S., 1981. Chemical control of cotton pests in Thailand. Trop. Pest Manage., 27 (4), 495-500

Wei C. S., Peng Z. J., Yang G. Q., Cao Y., Huang B. Z., Chen X., 1984. On the flower bug, Orius similis Zheng. Nat. Enemies of Insects, 6 (1), 32-40 
Yamamoto E., Nagai K., Nonaka K., 1981. Ecology and control of thrips infesting fruit vegetables. 1. Flight. Proc. Assoc. Plant Prot. Kyushu, 27, 98-99.
Yoshihara T., Kawai A., 1982. Parthenogenesis in Thrips palmi Karny. Proc. Assoc. Plant Prot. Kvushu, 28, 130-131. 